separation by other types of chromatography.

One of these is high-performance liquid chromatography (HPLC), a well-established lab workhorse. It uses a combination of solvents, pressure and matrix particle sizes to separate molecules on the basis of their retention times in a column packed with matrix. HPLC can separate a broad range of metabolites, including non-volatiles, and remains a favourite among metabolomics researchers.

Most advances in HPLC involve increases in the pressure applied and changes in matrix particle size. Ultra-performance liquid chromatography (UPLC), commercialized by Waters Corporation in Milford, Massachusetts, is becoming more widely used in the metabolomics community. It takes advantage of higher pressure (83 megapascals compared with 21 megapascals for HPLC) and smaller particles (less than 2 micrometres diameter compared with 3 micrometres for HPLC) to obtain faster separation times.

But like GC, HPLC has technical stumbling blocks. Reversed-phase HPLC (in which the stationary phase is non-polar) is often used for metabolomics analysis, but reversed-phase separation often fails with hydrophilic metabolites. These tend to be so water soluble that they interact poorly with the nonpolar bonding phase and are rapidly eluted, according to Phil Koerner, a senior technical manager from chromatography specialists Phenomenex in Torrance, California.

So in 2007, Phenomenex introduced the Luna HILIC column. "I like to refer to it as reverse reverse-phase chromatography," says Koerner. In the HILIC approach, the weak solvent, which is applied first, is a polar organic solvent (not water as in reversedphase HPLC), and the strong solvent, applied second, is water. This causes the order of elution to be completely reversed, with the most hydrophilic compounds being eluted last. Although Koerner acknowledges that the HILIC approach is not new, it was the need to separate hydrophilic metabolites on the large scale required by metabolomics that led Phenomenex and other companies, such as Waters and Tosoh Bioscience of Stuttgart, Ger- many, to start supplying a greater number and range of HILIC columns.

Capillary electrophoresis followed by MS (CE-MS) is not yet so popular with the metabolomics community as either GC or HPLC, but several developers are hoping to change this. "It can be very difficult to use this approach," acknowledges Ryuji Kanno, president of Human Metabolome Technologies based in Tokyo, Japan. This approach uses electrophoretic mobility to separate low-molecularweight ionic compounds that are difficult to separate by GC or HPLC. The company has been working closely with Agilent to develop optimized reagents and capillary columns, and is providing training with Agilent's CE-qTOF MS system to make the CE approach more accessible to metabolomics researchers, says Kanno.

Mass spectrometry is not the only method that can be used to detect metabolites once separated. Wishart and his colleagues recently compared MS and NMR to look at metabolites in cerebral spinal fluid ${ }^{2}$. They found little overlap in the metabolites detected by the two methods, and the conclusion was clear: "We do not have a single perfect metabolite detector," says Wishart.

MS and NMR each have their supporters. "One of the main strengths of NMR is that

HILIC columns are making the hunt for hydrophilic metabolites easier.

\title{
DARK MATTER
}

"There are a lot of small molecules that we do not even know about yet," says Arthur Castle, programme director for the Roadmap Metabolomics Technology development programme at the US National Institutes of Health in Bethesda, Maryland. Metabolomics has a good handle on analysing human primary metabolites, but when it comes to lipids, secondary metabolites, xenobiotics and the products of gut microflora, we are just scratching the surface, says Castle.

The problem is part technological, part informatics. Steven Fischer at Agilent Technologies in Santa Clara, California, points out that some compounds are not stable and undergo chemical transformation during separation. Researchers will probably still see these transformed molecules by mass spectrometry (MS), but they may be misidentified, highlighting the need for follow-up experiments. And this is where metabolomics has an advantage over the other 'omics'. "There is so much knowledge of biochemistry that when we find a potential biomarker or a new drug mechanism we already know a lot about it," says Michael Milburn, chief scientific officer at Metabolon in Durham, North Carolina.

Trent Northen, now at the Lawrence Berkeley Laboratory in Berkeley, California, and Oscar Yanes, working in Gary Siuzdak's lab at the Scripps Research Institute in La Jolla, California, may have developed a new way to get at some of these 'unknown' molecules with an ionization technique called nanostructure-initiator mass spectrometry (NIMS) ${ }^{4}$. The idea is to transfer a biomolecule into

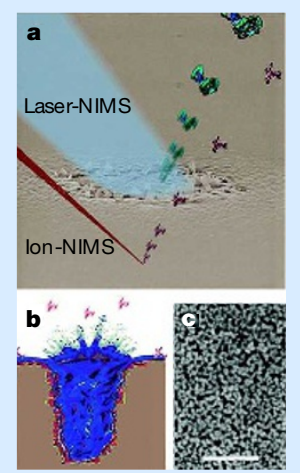

NIMS: nanostructured surface releases material. the gas phase from a nanostructured surface simply by making that surface disappear. "We came up with the idea of putting a wax underneath, so when the nanostructured surface was irradiated it would melt and vaporize, allowing the molecule to go into the gas phase," says Northen. They finally came up with a perfluorinate surface for the trapped initiator phase.

Siuzdak's group found that NIMS worked not only for proteins, but also for small molecules such as metabolites. "Perfluorinates do not ionize well, so it allows us to see things in the lower mass region where metabolites like to hang out," says Siuzdak.

Using perfluorinated materials may have another advantage for metabolomics. "As these are highly hydrophobic surfaces, we can apply very dirty complex samples, such as blood, for direct analysis," says Yanes. If you put a drop of urine or blood on the NIMS chip the metabolites will attach, but all the salts and other chemicals that normally interfere with MS stay in solution. Yanes is now exploiting this property to follow drug metabolism by looking at uptake in blood, clearance in urine and tissue localization.

For Siuzdak, exploring the 'unknown' metabolite world is an exciting prospect. "We are getting involved in an area where we don't know what the molecules' structures are or what they do, so it is really just a fantastic area for discovery." 